\title{
Implicaciones de la concepción de planificación por proyectos desde la perspectiva de actores educativos venezolanos
}

\author{
Implications of the Concept of Planning for Projects From the Perspective \\ of Venezuelan Educational Actors
}

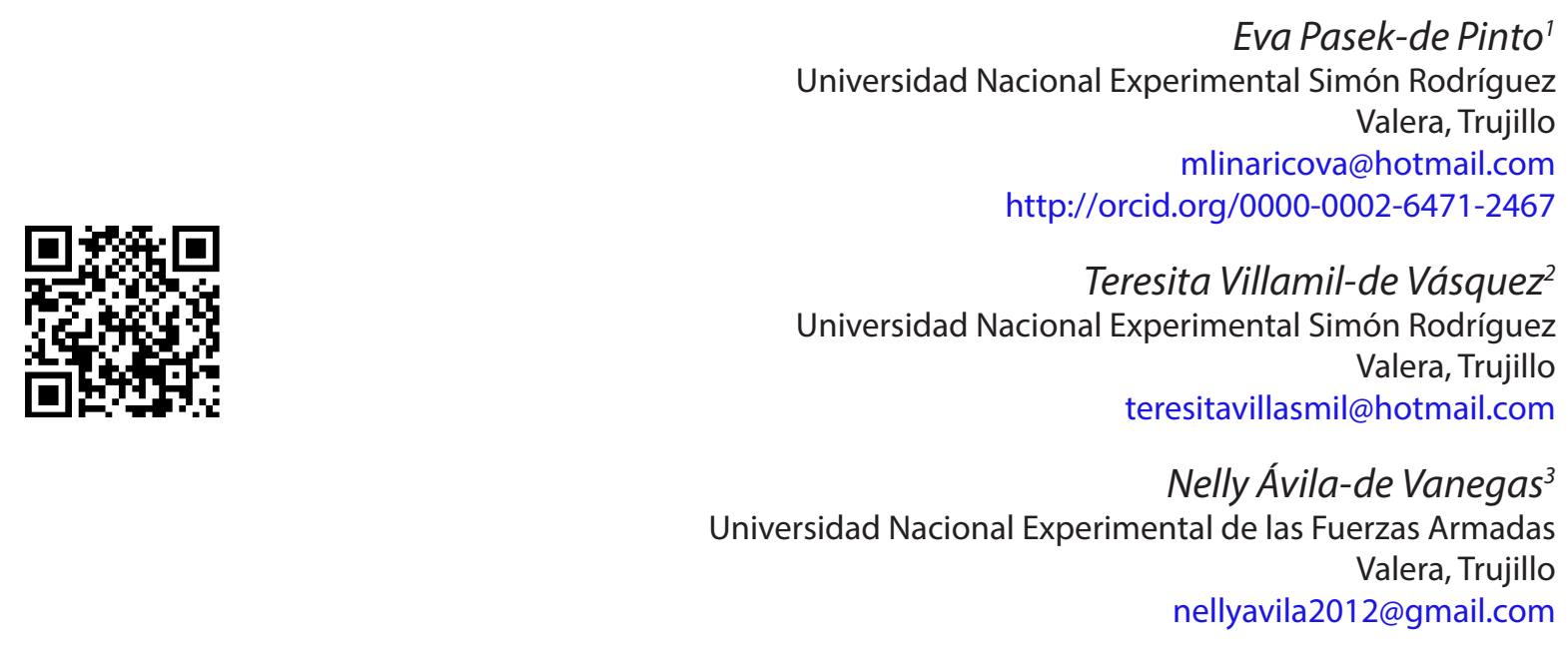

Recibido 122 de febrero de 2016 • Corregido 14 de noviembre de 2016 • Aceptado 18 de abril de 2017

Resumen: Este artículo proviene de una investigación que tuvo como objetivo derivar implicaciones educativas, epistemológicas, políticas y para el cambio a partir de las concepciones sobre planificación por proyectos que poseen los actores educativos. El estudio se abordó desde la corriente filosófica fenomenológica con una metodología cualitativa centrada en la entrevista. Siguiendo a Rodríguez, Gil y García (1999), la pesquisa se realizó en cuatro fases: la preparatoria, el trabajo de campo, el análisis y la informativa. Las entrevistas en profundidad fueron realizadas a tres directores, ocho docentes, cuatro representantes y dos estudiantes; se transcribieron, se organizó, codificó, categorizó por concepción, interpretó y trianguló la información recabada. Los resultados obtenidos indican que los actores educativos entienden la planificación por proyectos como visión, secuencia ordenada de pasos, acción colectiva, intención de logro, estrategia y meta. De estas ideas

\footnotetext{
${ }^{1}$ Doctora en Ciencias de la Educación. Docente jubilada de la Universidad Nacional Experimental Simón Rodríguez. Posee publicaciones en revistas nacionales e internacionales. Publicó un libro: "Didácticos. Ideas. Investigación. Educación" como autora-editora. Participa en el Programa de Estímulo a la Investigación e Innovación (PEII) en el nivel "C".

${ }^{2}$ Doctora en Ciencias de la Educación. Profesora jubilada de la Universidad Nacional Experimental Simón Rodríguez, Núcleo Valera. Posee publicaciones en revistas arbitradas e indexadas nacionales e internacionales. Es investigadora reconocida en el Programa de Estímulo a la Investigación e Innovación (PEII) en el nivel "B".

${ }^{3}$ Doctora en Educación. Actualmente profesora jubilada del Ministerio del Poder Popular para la Educación. Posee publicaciones en revistas arbitradas e indexadas nacionales. Es investigadora reconocida en el Programa de Estímulo a la Investigación e Innovación (PEII).
} 
doi: http://dx.doi.org/10.15359/ree.21-2.13

URL: http://www.una.ac.cr/educare

CORREO: educare@una.cr

se derivaron implicaciones educativas, epistemológicas, políticas y para el cambio. Se concluye que las concepciones de los actores educativos sobre la planificación por proyectos dificultan una educación para la democracia participativa, para la acción y el cambio, limitando la formación de la nueva ciudadanía responsable y cooperativa. Por eso, se sugiere formar para la participación al personal docente como responsables y garantes del proceso educativo.

Palabras claves: Planificación por proyectos; concepciones; implicaciones educativas; implicaciones políticas.

\begin{abstract}
This article is the result of a research project which aimed to infer educational, epistemological, political and transforming implications from the conceptions educational stakeholders have about planning for projects. The study was addressed from the phenomenological philosophical school perspective with a qualitative methodology based on interviews. Following Rodríguez et al. (1999), the research was conducted in four stages: the preparatory phase, the field work, the analysis, and the information phase. In-depth interviews were carried out with three principals, eight teachers, four representatives and two students. The information gathered was transcribed, organized, codified, categorized by concepts, interpreted and triangulated. The results indicated that educational stakeholders understand planning by projects like a vision, an orderly sequence of steps, a collective action, an intention of achievement, a strategy, and a goal. From these ideas derived educational, epistemological, and political implications for change. It is concluded that the conceptions of educational stakeholders on planning for projects render difficult an education for a participatory democracy, for action, and for change, limiting the formation of the new cooperative and responsible citizenship. Therefore it is suggested to train teachers for participation since they ensure the educational process.
\end{abstract}

Keywords: Planning for projects; conceptions; educational implications; political implications.

\title{
Introducción
}

La planificación estratégica se preceptúa participativa y democrática en la Constitución Nacional de la República Bolivariana de Venezuela del 20 de diciembre de 1999 (2000) en el artículo 299 para las actividades económicas; sin embargo, se ha trasladado a espacios sociales como la educación. Las instituciones de educación básica y media general organizan sus actividades por proyectos educativos que se asumen como participativos. El Proyecto Educativo Integral Comunitario (PEIC) (Ministerio del Poder Popular para la Educación, MPPE, 2007, 2012) es el que define las estrategias de gestión escolar, en el cual, sobre la base de una investigación de contexto, se planifica en conjunto el trabajo pedagógico de la institución y lo vincula con la comunidad. Así, en colectivo y por consenso se declara la visión, misión y objetivos de la institución, luego se aplica la matriz FODA (fortalezas, oportunidades, debilidades y amenazas) y se diseñan las estrategias de acción de tal manera que se aprovechan las fortalezas de la institución y las oportunidades que ofrece el entorno para atenuar las debilidades institucionales y eliminar las amenazas del entorno. De este proceso emerge el plan estratégico de la institución o PEIC, el cual se concreta en un plan de acción institucional y sirve de base a la planificación y 
evaluación. Las actividades educativas se organizan por medio de proyectos de aprendizaje. La educación por proyectos transforma el papel del estudiantado y personal docente, de tal forma que el primero se convierte en protagonista de su propio aprendizaje y el segundo se transforma en guía, acompañando y orientando el camino de la enseñanza. Se entiende que los proyectos generan un aprendizaje activo, coordinado, flexible, ya que se adaptan a las características de los distintos grupos de estudiantes y contextos socio-culturales.

Por eso los proyectos de aprendizaje, también participativos y democráticos, se deben construir en colectivo partiendo de un diagnóstico sobre los conocimientos, intereses y motivaciones de estudiantes e involucrando a la comunidad. Cada proyecto de aprendizaje debe estar vinculado con el PEIC, tratando de responder a los grandes problemas detectados en la institución.

Sin embargo, se observa una distancia notable entre el discurso y la acción, es decir, lo que se manifiesta verbalmente y lo que se hace. Ya en 2001, Pazos, representante del Ministerio de Salud Pública de Uruguay, expuso sus reflexiones y análisis sobre las dificultades que se presentan en la planificación de proyectos y que suelen limitar en gran medida los resultados que se desean alcanzar. Recientemente, Sarceda, Seijas, Fernández y Fouce (2015) explican que, si bien el trabajo por proyecto tiene más de 100 años, aún no es una práctica pedagógica extendida, lo que, según estas autoras, puede deberse al desconocimiento, falta de costumbre, miedo a no saber hacerlo bien, las propias características de la institución escolar, los resultados de aprendizaje "menos visibles" a corto plazo, etc.

De igual manera, en Venezuela la práctica educativa parece no coincidir con las consignas y las pautas del Estado para la educación, de modo especial en lo referente a la participación. Así se tiene que pocas veces se involucra en la planificación a la comunidad; también que mucho personal docente aún no planifica por proyectos, algunos no se vinculan con la comunidad, pocos relacionan los saberes académicos con los saberes populares; existe poca participación de la familia, escuela y comunidad en el proceso educativo, sumado a docentes con poca disposición para integrarse en las actividades lúdicas, recreativas, deportivas y culturales, tanto de la institución como de la comunidad.

Tomando en consideración que los proyectos de aprendizaje deben ser participativos, hay estudios que evidencian la escasa participación tanto del personal docentecomo dela comunidad y la secuela de sus concepciones al respecto. Así, Matos, Pasek y Rojas (2014) encontraron que el $52 \%$ de los padres y madres no asiste directamente a las actividades educativas de los niños y niñas; de estos, el 30\% aduce que trabaja y carece de tiempo disponible; el $22 \%$ son indiferentes a la participación. Del $48 \%$ que participa, $24 \%$ asiste a todas las actividades, el otro $24 \%$ solo cuando se realizan en la escuela. En una investigación anterior, Pasek de Pinto, Ávila de Vanegas y Matos de Rojas (2015) hallaron que la participación social es entendida como: integración (20\%), involucramiento (13\%), colaboración (13\%) y opinión (27\%). De ello se infirió que solo $33 \%$ de los actores concibe la participación como un compromiso de actuar y, además, un 73\% reconoció que se abstiene de participar. 
doi: http://dx.doi.org/10.15359/ree.21-2.13

URL: http://www.una.ac.cr/educare

CORREO: educare@una.cr

Por tal motivo, esta investigación tuvo como objetivo derivar implicaciones educativas, epistemológicas, políticas y para el cambio de las concepciones sobre planificación por proyectos que poseen los actores educativos. Su importancia radica en que las creencias y concepciones de las personas orientan sus acciones, de manera que la concepción que posean sobre la planificación por proyectos determina su actuar educativo en correspondencia o no con los lineamientos educativos preceptuados por el MPPE (2007) en el Currículo Nacional Bolivariano, asumiendo o no un sentido de pertenencia, compromiso e interés en el proceso educativo.

Metodológicamente el estudio se abordó por medio de una metódica cualitativa centrada en la entrevista. Tomando como base el proceso de investigación sugerido por Rodríguez et al. (1999), el desarrollo de la investigación consistió en recabar la información, analizarla e interpretarla y elaborar el informe.

\section{Fundamentación teórica}

\section{La planificación estratégica}

Aplicar la planificación ha contribuido a que existan variadas definiciones. Molins (2007, p. 20) recoge, entre otras, las siguientes: Para Ahumada, "la planificación ... es una metodología para la toma de decisiones". Chadwick señala que "La planificación es un proceso de reflexión y acción humana basada en la reflexión en realidad, premeditación o reflexión para el futuro". Por su parte, Giordani entiende la planificación "como un proceso social que supone elementos derivados de dos vertientes diferentes, una de tipo histórico-concreto y otra de tipo abstracto formal". Las definiciones recabadas por Molins (2007), no obstante que parecen diferentes, más bien se complementan en los aspectos metodológicos, sociales y administrativos de la planificación.

Por su parte, Corredor (2010) expresa que "planificar, en su sentido más lato, es aproximarse al futuro, estando en el presente, para configurar modelos o escenarios a la medida de nuestras ideas" (p. 1). Como modo de aproximación al futuro, Molins (2007) diferencia tres modalidades de planificación: normativa, estratégica y reiterativa o iterativa; Malagón (2005) indica la planificación estratégica, situacional, clásica, de sistemas, para el desarrollo y prospectiva; y, finalmente, Corredor (2010), planificación administrativa, normativa y estratégica. Dado que la planificación por proyectos, objeto de este estudio, se sustenta en la planificación estratégica, esta se profundiza a continuación.

La planificación estratégica, según Corredor (2010), contiene dos elementos de análisis: planificación y estrategia. El autor señala que, si bien la planificación estratégica nace en el campo militar, se aplica en diferentes ámbitos. En ella, la estrategia formula principios que moverán el proceso para aventajar al ente competidor; la táctica los desagrega en acciones concretas y con objetivos inmediatos. Se entiende, entonces, la estrategia como un accionar; por lo que, según Morin $(2000,2007)$, se opone a la palabra programa, en tanto algo predeterminado e invariable 
que basta aplicar. Pero la acción es una decisión, una elección y es, también, una apuesta. Un programa funciona para el logro de los objetivos cuando las circunstancias no cambian, pero, el mundo cambiante de hoy y las transformaciones y reformas educativas actuales exigen estrategias, las cuales se preparan, integran lo nuevo y enriquecen sus acciones. Sin embargo, de acuerdo con Morin (2007), para que una estrategia sea llevada a cabo por una organización, requiere que esta "sea capaz de tratar a los elementos capaces de contribuir a la elaboración y al desarrollo de la estrategia" (p. 127), y no una concebida para obedecer a la programación. De ahí la importancia de la planificación estratégica.

Volviendo a Corredor (2010) encontramos que la planificación estratégica "propone, estudia y conduce las acciones con una perspectiva de cambios sustanciales de la situación" ( $p$. 65). Para Armijo (2009), la planificación estratégica:

Es una herramienta de gestión que permite apoyar la toma de decisiones de las organizaciones en torno al quehacer actual y al camino que deben recorrer en el futuro para adecuarse a los cambios y a las demandas que les impone el entorno y lograr la mayor eficiencia, eficacia, calidad en los bienes y servicios que se proveen. (p. 5)

La misma autora afirma que se caracteriza por su orientación a la acción mediante estrategias que facilitan el logro de los objetivos planificados. En este sentido, se constituye en "una herramienta clave para la toma de decisiones de las instituciones públicas" (p. 6), puesto que el hecho de partir de un diagnóstico situacional le facilita decidir las acciones futuras con la finalidad de alcanzar la situación deseada. Pero esto implica que, ante cambios en las condiciones o situaciones, se requiere valorar la efectividad de las estrategias implementadas para, en caso de ser necesario, cambiar el rumbo. Por eso, la autora mencionada asevera que "cuando hablamos de planificación estratégica nos estamos refiriendo a las grandes decisiones, al establecimiento de los Objetivos Estratégicos que permiten materializar la Misión y la Visión"(Armijo, 2009, p. 8).

En esta línea de pensamiento y desde una perspectiva educativa, SEP (2009) explica que una planeación estratégica:

Es participativa cuando en la escuela se involucra a los alumnos, padres de familia, maestros, director y, como apoyos externos, al supervisor, jefe de sector y/o de enseñanza. Su diseño, ejecución y seguimiento es responsabilidad de todos ellos y muestra los deseos de mejora y las formas que, desde su punto de vista, son las idóneas para obtenerla, dando respuesta a la capacidad de decisión basada en el conocimiento de su contexto. (pp. 66-67)

En este sentido, la planificación por proyectos es un componente central del sistema educativo venezolano, de las actividades profesionales y de la vida cotidiana. Alude a los procesos que escuela, familia y comunidad ejecutan para superar los obstáculos; por lo cual, amerita una 
doi: http://dx.doi.org/10.15359/ree.21-2.13

URL: http://www.una.ac.cr/educare

CORREO: educare@una.cr

concepción holística que oriente la comprensión contextual del proceso de planificación por proyectos, de sus protagonistas y de sus contextos. Tal como se puede observar en las ideas básicas para la elaboración del PEIC que establece el MPPE (2012):

- Es la metodología de gestión escolar que concreta la tríada familia-instituciones educativas-comunidad.

- Revela la planificación educativa desde su carácter estratégico.

- Es favorecedor en su elaboración de habilidades para la investigación de las y [sic] los participantes y corresponsables del proceso educativo.

- Revela un carácter integral e integro [sic] debido a las relaciones que se establecen entre sus fases, al interior de las mismas, devela un continuo desarrollo cíclico que en espiral describe avances en sucesivas construcciones hacia un resultado cualitativo superior.

- Favorecedor de intercambio de saberes y construcción de conocimientos, donde los referentes teórico-prácticos son pertinentes socioculturalmente. (p. 6).

\section{La planificación por proyectos y los proyectos educativos}

De acuerdo con Ander-Egg y Aguilar (2000), la planificación tiene diferentes niveles de concreción, que van desde el plan hasta las tareas. Así, el plan está conformado por programas, los programas, a su vez, por proyectos, los proyectos por actividades y estas por tareas. Luego, en términos generales, un proyecto constituye "un conjunto de actividades concretas, interrelacionadas y coordinadas entre sí, que se realizan con el fin de producir determinados bienes y servicios capaces de satisfacer necesidades o resolver problemas"(p. 16). Así entendidos, los proyectos constituyen modos de planificación y configuran el medio de acción más idóneo para dirigir los procesos sociales, incorporar cambios y lograr transformaciones; su propósito fundamental es prever los acontecimientos futuros.

Según Flores y Agudelo (2006), SEP (2009), en el ámbito educativo la planificación por proyectos favorece la interacción donde los niños, niñas y jóvenes descubren el sentido de la vida como ciudadanos participativos, responsables, cooperativos y democráticos, además de ofrecer espacios para la transformación del individuo a partir de la producción y el desarrollo endógeno, el quehacer comunitario, la formación integral, la creatividad, la promoción de la salud y el respeto por la vida, las innovaciones pedagógicas, la comunicación, el uso y desarrollo de las tecnologías, la participación y el trabajo en equipo, la consolidación de los principios democráticos y de la paz. 
En el sistema educativo bolivariano y conforme con el MPPE (2007), la institución educativa debe promover:

1. La formación de un ser humano integral social, solidario, crítico, creativo y autodidacta.

2. El fomento de los Derechos Humanos y el ejercicio de la cultura de la paz.

3. La participación democrática, protagónica y corresponsable, en igualdad de derechos, deberes y condiciones. (p. 22)

Para ello, el mismo documento establece que la organización y estructuración de los diferentes elementos que condicionan el proceso de aprendizaje se deben realizar por medio de la clase, el proyecto y el plan integral. En este contexto, los proyectos educativos se entienden como medios organizadores y se definen como sigue:

Proyecto educativo integral comunitario (PEIC): "es el proyecto que define las estrategias de gestión escolar. Sobre la base de investigación de contexto planea en conjunto el trabajo pedagógico de la institución y lo vincula con la comunidad"(MPPE, 2007, p. 66). Adicionalmente, el MPPE (2012) señala que es la metodología de gestión escolar que incluye la familia, las instituciones educativas y la comunidad; evidencia el carácter estratégico de la planificación en educación y favorece el intercambio de saberes y construcción de conocimientos, donde los referentes teórico-prácticos poseen pertinencia sociocultural.

En ese sentido, el PEIC se puede considerar como una acción estratégica que centra su interés en la planificación por proyectos, ya que permite articular el quehacer educativo en estrecha vinculación y participación con todos los involucrados a la escuela como alternativa para su transformación. Sería el primer paso para la participación de los estudiantes en el acontecer comunitario y, de esta manera, lograr articular la formación, la investigación y la proyección social.

Proyecto de aprendizaje (PA): Según el MPPE (2007):

Se centra en la investigación-acción, [su] escenario es construido por los actores comprometidos, creando las condiciones que permitan el trabajo cooperativo sobre la base de situaciones reales de la vida diaria y con acciones que impliquen prácticas y desarrollos que afectan al ser humano en sus condiciones de vida, dándole sentido a lo que este aprende. (p.66)

Es de hacer notar que la investigación-acción, según Kemmis (1996, p. 52), "es una forma de indagación autorreflexiva, emprendida por participantes en situaciones sociales con la 
doi: http://dx.doi.org/10.15359/ree.21-2.13

URL: http://www.una.ac.cr/educare

CORREO: educare@una.cr

finalidad de mejorar su práctica social o educativa, así como la comprensión de las mismas". De igual manera, Elliott (1996, p. 28), la define como:"El estudio de una situación social con el fin de mejorar la calidad de acción dentro de la misma".

En ese orden de ideas, el proyecto de aprendizaje "está concebido como un proceso estratégico que orienta el desarrollo de aprendizajes desde el diálogo de saberes y la construcción de conocimientos" (MPPE, 2012, p. 13), puesto que es construido en forma colectiva entre maestros, maestras y estudiantes. De allí que guarda estrecha relación con el proyecto educativo integral comunitario, por cuanto aproxima a docentes y estudiantes a la reflexión y a la toma de conciencia acerca de los problemas sociales y los valores que orientan la sociedad.

Proyecto de desarrollo endógeno: Según el MPPE (2007):

Tiene como fin la participación de manera integrada de todos los actores en el proceso educativo (maestros, maestras, estudiantes y familia), quienes a partir de la realidad implementan diferentes acciones para su transformación; es decir, impulsan a las [sic] y los jóvenes desde su propio contexto, tomando en cuenta los aspectos socio-ambientales de la comunidad, de tal forma que contribuyan con el desarrollo endógeno local, regional, nacional, latinoamericano, caribeño y mundial. (p. 66)

Al respecto, Castellano (2006) expone que para calificar como "endógeno" un proyecto, se deben considerar criterios relacionados con la "participación relativa local en la decisión, el capital, la gerencia, la mano de obra y los insumos" (p. 83).

Plan integral: "es una forma para planificar componentes que no se abordan en los proyectos de aprendizaje. En este, se deben tomar en cuenta los pilares y los ejes integradores" (MPPE, 2007, p. 67). El mismo documento expone que los pilares son: aprender a crear, aprender a convivir y participar, aprender a valorar y aprender a reflexionar. $Y$, los ejes integradores: ambiente y salud integral, interculturalidad, las tecnologías de la información y comunicación (TIC) y trabajo liberador.

\section{Metodología}

Por su naturaleza, constituye un estudio cualitativo. La investigación cualitativa aporta datos descriptivos como las propias palabras de las personas, habladas o escritas, así como los comportamientos (Taylor y Bogdan, 1992).

Dado que esta indagación forma parte de un proyecto grupal en ejecución más amplio, para obtener la información se consideró la misma muestra de informantes del estudio previo. Para seleccionar la muestra, conversamos con los 30 docentes de la institución, les informamos del propósito y les propusimos ser partícipes de la investigación. En un segundo encuentro, hablamos con el personal docente que se mostró más receptivos; de este, 11 aceptaron voluntariamente formar 
parte del estudio. A representantes y estudiantes les abordamos durante una reunión y consultamos a quienes accedieron a conceder la entrevista. En consecuencia, son 17 personas: "[3 directores (D1, D2, D3)], 3 maestras (DOC1, DOC2, DOC3), 5 profesores por hora (PH1, PH2, PH3, PH4, PH5), 4 representantes (REP1, REP2, REP3, REP4) y 2 estudiantes (E1, E2)" (Pasek de Pinto et al., 2015, p. 107). Cabe aclarar que se distingue entre maestros, maestras y profesores, profesoras por hora, ya que el primer grupo tiene a su cargo un grupo de estudiantes durante todos los días de la semana y cumple un horario completo; el segundo dicta determinadas asignaturas con un número de horas específicas, cumple horas en una sección y, al terminar, pasa a otra o se retiran de la escuela. La mayoría labora en varias instituciones, lo que limita su pertenencia, compromiso y participación en el plantel. No obstante, la totalidad debe planificar y ejercer su labor por medio de proyectos de aprendizaje.

Para recabar la información se utilizó la entrevista cualitativa, flexible y dinámica; esta permite profundizar en el fenómeno que se indaga desde la perspectiva del otro ser. Así, adopta la forma de un diálogo coloquial a la vez que facilita centrar la atención sobre una temática específica de interés para quien investiga (Taylor y Bogdan, 1992). Las entrevistas tuvieron una duración promedio de 30 minutos y fueron grabadas con el permiso de cada informante; al estudiantado se consultó en compañía de su representante, aprovechando para reunirse con ambas partes. Los lugares elegidos para la entrevista fueron la oficina (directores y directoras), el aula (docentes), la cantina escolar o cafetín (docentes, estudiantes-representantes), un banco en el patio de recreo (estudiantes-representantes). El instrumento consistió en una guía de entrevista contentiva de 1 pregunta básica: ¿Qué es para usted la planificación por proyectos?, y se permitió la repregunta para aclarar o ampliar las respuestas.

Para el análisis se siguió a Rodríguez et al. (1999), quienes establecen un proceso general de análisis que va desde la transcripción a la interpretación de la información generada. Ya transcrita, la entrevista se entregó a cada entrevistado para su revisión y conformidad. Luego la información se organizó, codificó, categorizó, interpretó y, para validarla, se trianguló considerando como fuentes la visión de los actores educativos, la conceptualización de autorías teóricas y la interpretación de las autoras. El análisis se realizó por medio de la categorización, ya que cada categoría soporta un significado o tipo de significados. Finalmente, se elaboró el informe de la investigación.

\section{Resultados}

Considerando el objetivo del estudio, en los resultados se presentan las concepciones que poseen los actores educativos sobre la planificación por proyectos en la forma de categorías. Posteriormente, desde estas se derivaron las implicaciones objeto de indagación. Así, se encontró que la planificación por proyectos es vista como: visión, secuencia ordenada de pasos, acción colectiva, intención de logro, estrategia y meta. En lo que sigue se presenta cada categoría con la evidencia oral de los actores, el argumento teórico y su correspondencia o no con lo preceptuado por el MPPE (2007). 
doi: http://dx.doi.org/10.15359/ree.21-2.13

URL: http://www.una.ac.cr/educare

CORREO: educare@una.cr

\section{Visión}

Algunos actores educativos entrevistados conciben la planificación por proyectos como una visión. Esta concepción se reflejó en las siguientes expresiones: D1 indicó: “Es una visión de lo que se quiere lograr. Es recopilar una información para ejecutar algo que se quiere". Para PH2: "El proyecto es una perspectiva o visión de lograr lo que uno se propone, incluyendo el qué, cómo y cuándo lo voy a hacer". Asimismo, PH4 señaló que: "Un proyecto es una visión a futuro de actividades a realizar para conseguir algo basado en una realidad social, de la cual, salen unas variables y las que resulten, se les dará respuestas a través de actividades que conforman un conjunto".

Las expresiones precedentes de los actores educativos revelan dos aspectos. El primero lo constituye el hecho que ninguno habló de "planificación por proyectos", sino que, obviando la palabra planificación, definieron proyecto. El segundo aspecto fue la relación teleológica entre visión y acción, evidente en sus frases: "para ejecutar algo" (D1); "lograr lo que uno se propone, incluyendo el qué, cómo y cuándo lo voy a hacer" ( $\mathrm{PH} 2)$; "actividades a realizar para conseguir algo" (PH4). Es decir, el proyecto es una visión que nos lleva al logro de algo deseado.

Veamos ahora quées una visión. Para Senge (1995),"es una imagen del futuro que deseamos crear, descrita en tiempo presente como si sucediera ahora. Una proclama de visión muestra a dónde queremos ir y cómo seremos cuando lleguemos allí" (p. 314); y en Senge (1993), la visión "es el qué, la imagen del futuro que procuramos crear" (p. 283). En este sentido, la visión infunde rumbo al futuro ayudando a fijar metas que impulsan las aspiraciones de una organización al logro. Sin embargo, dado que la visión es lo que deseamos alcanzar ejecutando el plan, resulta claro que la planificación por proyecto o el proyecto no son una visión. En correspondencia con esto último, Armijo (2009) dice que hablar de planificación estratégica nos refiere a las decisiones, a implantar los objetivos estratégicos que permiten cristalizar la misión y la visión.

Por otro lado, Ander-Egg y Aguilar (2000) en su definición de proyecto señalan que este contiene actividades que se concretan en acciones y tareas; su ejecución llevará al logro de la visión. Luego, al comparar las definiciones de los autores con la concepción del grupo informante, podemos visualizar cierta confusión de los actores educativos, pues, si bien un proyecto incluye acciones, la visión es el futuro que perseguimos con ellas. En consecuencia, el proyecto no es la visión, sino el modo, la acción para lograrla y la visión constituye un componente de la planificación por proyecto.

Esto implica que la concepción del proyecto como visión no necesariamente lleva a su logro, son las acciones las que conducen a su fin. Además, Senge (1993) plantea que para que la visión actúe como un motor de las acciones debe ser compartida. Contradiciendo esto, la visión que orienta las escuelas bolivarianas fue establecida por el Ministerio de Educación y Deportes (2004). 


\section{Secuencia ordenada de pasos}

En esta categoría se incluyeron las siguientes expresiones: PH2, dijo: "La planificación por proyectos son fases para lograr metas, en las cuales se toman en cuenta las propuestas de los involucrados en el proyecto"; para DOC3, la "Planificación por proyectos es seguir unos pasos para lograr todo lo plasmado en el proyecto", y, DOC2 expresó: "La planificación por proyectos es la secuencia y el orden de lo que se quiere lograr en los proyectos". En las locuciones anteriores vemos que la planificación por proyectos la entienden algunos actores educativos como fases, pasos, secuencia ordenada, nociones que implican el concepto de proceso, pero de manera implícita, puesto que ninguno se refirió a ella como tal.

Al analizar las concepciones encontramos los siguientes aspectos. En principio, en todas las concepciones se encuentra tácita la idea de actividades que se realizan. Aunado a esto, por una parte, la planificación por proyectos es entendida como fases para lograr un fin; por la otra, se habla de "seguir unos pasos", o "secuencia y orden" lo que refiere a etapa. Por eso, aquí cabe señalar la precisión que realizan Collerette y Delisle (2001), al explicar que la noción de etapa contiene una separación cronológica entre las actividades que supone que estas se llevan a cabo una a una, mientras que la noción de fase implica que se pueden realizar de forma concurrente. En ese orden de ideas, las expresiones "seguir unos pasos" $y$ "secuencia y orden" hacen referencia a etapas programadas que se ejecutan en el orden establecido y que, en consecuencia, solo pueden ocurrir en ámbitos libres de cambio e incertidumbre por lo cual no requiere ajustes ni modificaciones, lo que resta flexibilidad al plan/proyecto.

Frente a tal concepción, Ander-Egg (2007) declara que la planificación es un proceso, es decir, es una actividad continua y unitaria ... que implica un reajuste permanente entre medios, actividades y fines $y$, sobre todo, de caminos y procedimientos a través de los cuales se pretende incidir sobre algunos aspectos de la realidad. (p. 27)

Luego, los actores educativos tienen claro que se pretende lograr un fin por medio del proyecto, que los proyectos involucran actividades; sin embargo, poseen una noción del plan/ proyecto como algo terminado que se debe seguir en el orden de los pasos establecidos. Esto contradice el objetivo del MPPE (2007) de "generar un proceso educativo que permita desde la construcción de los conocimientos, caracterizar, reflexionar y analizar la realidad para transformarla" (p. 55), ya que la transformación involucra ajustes y reajustes, teniendo en cuenta los actores sociales y los factores del escenario donde se desarrolla el proyecto.

Otro error de conceptualización en el cual incurren los actores educativos consiste en la confusión del proceso de planificación con alguna de sus fases, específicamente con las fases de organización y ejecución. Así, la planificación del aprendizaje por proyectos fue concebida por los actores entrevistados como organización, la cual se refleja en los siguientes términos: 
doi: http://dx.doi.org/10.15359/ree.21-2.13

URL: http://www.una.ac.cr/educare

CORREO: educare@una.cr

Para E2 "La planificación del aprendizaje por proyectos es la organización de las funciones para dar cumplimiento a los proyectos"; también, PH3 expresó: "Planificación por proyectos es un conjunto de técnicas y estrategias. Es la acción de organizar un conjunto de actividades previstas para dar solución a una problemática".

En las voces precedentes, se observa que los actores educativos conciben la planificación por proyectos como la organización de actividades a seguir para dar cumplimiento y solución a una problemática abordada en los proyectos. En consecuencia, tal concepción contradice lo señalado por Rossi y Grimberg (2005), para quienes organización "es la integración de un conjunto de unidades para la consecución de un fin" (p. 70); igualmente, contradice a Molins (2007), quien explica que en la "etapa de la instrumentación o implementación, se preparan las condiciones y se obtendrán los medios que permitan lograr la mayor eficacia en la aplicación del plan" (p. 30). En suma, la organización supone el arreglo de las acciones que serán ejecutadas para alcanzar las metas de la mejor forma posible.

Por otro lado, la concepción de la planificación por proyectos como ejecución de algo previsto se hizo evidente en las palabras de: REP4, quien dijo:"Un proyecto es algo que se va a ejecutar, algo que se necesita en la escuela y la comunidad"; y de DOC1, cuando manifestó: "La planificación por proyecto es la ejecución de los proyectos, es la puesta en práctica de lo planificado".

Las definiciones involucran la idea de planificación por proyectos como ejecución o puesta en práctica. Al analizar esta noción, se destacan dos errores: el primero consiste en el hecho de que la ejecución es una fase de la planificación por proyectos, pero no el proyecto. El segundo error está en la confusión implícita entre acción y ejecución, distinción que ofrecen Collerette y Delisle (2001):

La ejecución se refiere a la fase en que se procede a la implantación de las soluciones elegidas y elaboradas en las fases precedentes. La acción se refiere a toda medida que se tome para influir sobre el sistema social dentro del marco de la intervención. (p. 63)

Luego, aunque el proyecto esté configurado por un conjunto de actividades que se desagregan en tareas o acciones para ser realizadas (Ander-Egg, 2007), su ejecución conforma una fase del proceso de planificación por proyectos. En palabras de Flores y Agudelo (2006, p. 37): la ejecución "implica poner en marcha el PEIC"; o de Pérez (2000): "la ejecución implica poner en práctica el proyecto y prestar atención a su desarrollo, seguimiento y control" (p. 105).

Cabe agregar aquí a D1, quien indicó que la planificación por proyectos "Es una visión de lo que se quiere lograr; es recopilar una información para ejecutar algo que se quiere", pues, aunque expresó la definición como una visión, la completó como ejecución, evidenciando un error adicional al asumir la ejecución como la búsqueda de información, fase de la planificación que corresponde al diagnóstico previo, pero no define el proyecto. Recapitulando, lo antes expuesto revela cierto 
desconocimiento de estos actores puesto que, primero, confunden acciones con diferentes fases de un proceso; acciones que, perteneciendo al campo de la planificación, designan aspectos o fases distintas del proceso, y segundo, tales aspectos no definen la planificación por proyectos.

\section{Acción colectiva}

La planificación por proyectos es entendida por algunos actores como una acción grupal, un hacer colectivo, noción evidente en las expresiones: REP1 expresó: "Un proyecto es cuando entre varias personas hacen algo para beneficio de la comunidad donde viven"; asimismo, E1 señaló: "Para mí un proyecto es un trabajo, cuando me dicen: vamos a hacer algo, eso es un proyecto"; también, REP4 indicó: "La planificación por proyectos viene siendo la reunión de docentes y estudiantes para los pasos que se van a seguir en la ejecución de los proyectos".

Primero, es importante señalar que en la concepción de planificación por proyectos no se menciona la planificación, sino directamente los proyectos; además, que pertenece a dos representantes y un estudiante. Es decir, no fue expuesta por ningún docente, teniendo presente que son estos (maestras y profesores por hora) quienes deben planificar sus actividades mediante proyectos.

En segundo lugar, estos actores indican que el proyecto involucra una acción, es un hacer. Esto se corresponde con lo que dice Corredor (2010), al definir la acción como "el modo de hacer, es decir, el proceso práctico. La acción significa movimiento en una dirección acertada, una trayectoria de la cual depende el resultado deseado" (p. 83). Igualmente, con Ander-Egg (2007), cuando expresa que la planificación es una actividad eminentemente práctica, pues se planifica para la acción; aunado al hecho de que Ander-Egg y Aguilar (2000), al definir el proyecto como conjunto de actividades concretas,_establecen que planificar por proyectos involucra la acción.

En tercer lugar, todas las manifestaciones orales tienen la idea de un hacer en colectivo, no individual: "entre varias personas", "vamos a", "reunión de", al igual que en la concepción de PH2: "La planificación por proyectos son fases para lograr metas, en las cuales se toman en cuenta las propuestas de los involucrados en el proyecto", quien, aunque la entiende como fase, explicita que existen unos "involucrados". Es decir, para estos actores la planificación por proyectos es una acción participativa, lo que guarda correspondencia con la definición que plantea el MPPE (2007), al especificar que los proyectos involucran un proceso activo y participativo, especialmente el proyecto integral comunitario (PEIC), en el cual se planifica el trabajo pedagógico de la institución en unión íntima con la comunidad.

En este punto es de hacer notar que al preguntar sobre la elaboración del PEIC en la institución, se obtuvieron las siguientes respuestas: La docente DOC1 manifestó: “Este año no hemos tenido el proyecto institucional", por su parte, PH2 admitió: "Pues no tengo conocimiento del PEIC de la escuela", igualmente, PH4 dijo:"Del proyecto institucional: no tengo conocimiento", sumado a estos, E1 señaló: "En cuanto al proyecto institucional, no sé nada". 
doi: http://dx.doi.org/10.15359/ree.21-2.13

URL: http://www.una.ac.cr/educare

CORREO: educare@una.cr

Enlasfrasesanteriores, docentes, profesoradoyalumnadomanifestaronsudesconocimiento con respecto al PEIC. De este desconocimiento se puede inferir que los actores educativos no participaron en su elaboración, lo que contradice planteamientos como el de Rossi y Grinberg (2005), quienes afirman que "la elaboración del PEIC, corresponde a los diferentes miembros de la comunidad escolar. La participación de todos ellos en una acción conjunta es el fundamento para que el instrumento refleje posturas consensuadas y asumidas conscientemente" (p. 158), resaltan que personal directivo, docente, estudiantes, padres, madres y personal no docente deberían participar en la construcción del PEIC. De igual manera, contraviene lo establecido por el MPPE (2007), ya mencionado. El PEIC, como modo de planificación por proyectos, es el proyecto institucional donde se definen las estrategias de gestión escolar, debe involucrar a los actores sociales vinculados con la escuela mediante un proceso activo y participativo e incluir los otros tipos de proyectos.

En síntesis, aunque el proyecto en sí mismo no es una acción, sí comprende actividades y acciones y estas deben planificarse y realizarse en colectivo. Por otra parte, la planificación por proyecto es una actividad práctica que debe involucrar a todos los actores educativos y sociales vinculados con la institución educativa y aparentemente no es así.

\section{Intención de logro}

Bajo esta categoría se ubicaron diversas expresiones en las cuales subyace la idea de intención de acción para un logro: plan, documento, propuesta y aspiración. En cuanto a la concepción de planificación por proyecto como un plan, el directivo D2 indicó: "el proyecto es para mí como un plan, es algo, son propuestas a corto, mediano y largo plazo factibles de lograr", y, por su parte PH1 dijo: "La planificación por proyecto es establecer un objetivo por medio de etapas y diversas estrategias a aplicar y a ser logradas a corto, mediano y largo plazo"; o como un documento: DOC3 señaló que:"el proyecto es un documento para llevar a cabo unos pasos a seguir, es la manera de trabajar ordenadamente". También la planificación por proyectos entendida como una propuesta: para REP2: “El proyecto es una propuesta para beneficio de la escuela y la comunidad"; en cuanto a REP3, dijo: "Un proyecto es una solicitud de hacer algo para beneficio de la escuela, para solucionar un problema", asimismo, E2 expresó: "Un proyecto es una propuesta para realizar algo a fin de mejorar el funcionamiento de la escuela"; y DOC2, señaló:"Un proyecto es una aspiración que uno quiere lograr, que siempre se hace con un fin a futuro".

Veamos ahora algunas distinciones. Ander-Egg (2007) diferencia entre la planificación como elaboración de planes, programas y proyectos y la planificación como proceso y estrategia, distinción en la cual asocia la planificación como elaboración de proyectos con el diseño de un documento, mientras que la planificación como proceso y estrategia posee significado de 
acción. Esta misma divergencia la presenta Corredor (2010) cuando aclara que planificación refiere a un proceso para lograr objetivos; por el contrario, planeación es hacer planes. En este mismo orden de ideas sobre hacer un plan, Barrera (2005) dice:

La elaboración de un plan constituye el documento formal en el cual se expresa de manera organizada y amplia lo relacionado con posibilidades, inquietudes, opciones y aspiraciones que recogen de manera concreta, el qué se va a hacer, con los demás aspectos de tal propósito: quiénes, recursos, tareas y propósitos. (p. 14)

Aunado a lo anterior, el MPPE (2007) establece que el Plan Integral "es una forma para planificar componentes que no se abordan en los proyectos de aprendizaje" (p. 67), lo que significa que un plan no es lo mismo que un proyecto, aunque ambos constituyan formas de planificar.

Al analizar las discrepancias, encontramos que un plan precisa acciones con miras a lograr objetivos, fija responsabilidades y asigna recursos para un período dado y se puede objetivar en un documento como producto y representación del proceso de planificación. Además, encarna unas proposiciones o ideas que se ofrecen o presentan para lograr un fin o aspiración de logro. Luego, al trasladar estas divergencias a la definición de planificación por proyectos, encontramos cierta confusión o desconocimiento por parte de los sujetos, puesto que, si bien el plan puede ser un documento, la planificación por proyectos constituye un proceso y no un documento, aunque se entregue como tal. Adicionalmente, un proyecto contiene las acciones y tareas a realizar; lo que es contrario a una propuesta en el sentido de ideas, intención o aspiración de acción.

En síntesis, la planificación por proyectos se puede objetivar en forma de documento, pero no es el documento; es una forma de planificación con un nivel concreto de actividades que puede incluir una propuesta y las aspiraciones de un grupo humano, pero no es en sí misma una propuesta ni una aspiración.

\section{Estrategia}

La planificación por proyectos es concebida como una estrategia, pensar que se evidencia en la expresión de PH3, quien expuso: "El proyecto es una estrategia que me va a permitir dar solución a un problema o problemática; es la acción de organizar un conjunto de actividades previstas para dar solución a una problemática" Y agregó: "Yo no tengo conocimiento de la planificación por proyectos porque yo planifico con el programa". Al analizar esta noción vemos que refiere a una acción sobre la realidad con el fin de mejorarla. Además, notemos cuatro aspectos fundamentales: 1 . un proyecto es estrategia; 2 . un proyecto es acción de organizar actividades, 3. un proyecto es estrategia que permite solucionar problemas; y, 4. la distinción entre proyecto y programa. 
doi: http://dx.doi.org/10.15359/ree.21-2.13

URL: http://www.una.ac.cr/educare

CORREO: educare@una.cr

Con respecto al segundo aspecto, previamente vimos que la organización es un paso del proceso de planificar por proyectos y supone el arreglo de las acciones a ejecutar para alcanzar las metas previstas, pero, no define la planificación por proyectos como proceso ni al proyecto en tanto conjunto de tareas y acciones; tampoco define una estrategia. En consecuencia, es una visión errónea sobre la planificación por proyectos.

Veamos ahora algunas definiciones de estrategia para comparar luego. Molins (2007) señala que, en sentido restringido, "estrategia [es] el conjunto de líneas generales de acción a ejecutar con el propósito de lograr, en el objeto, el futuro propuesto por el sujeto planificador" (p. 48). Es decir, el autor equipara estrategia con acción. Por su parte, Morin (2007) explica que la palabra estrategia se opone a la palabra programa. En su propia voz:

La acción es estrategia. La palabra estrategia no designa a un programa predeterminado que baste aplicar ne variatur en el tiempo. La estrategia permite, a partir de una decisión inicial, imaginar un cierto número de escenarios para la acción, escenarios que podrán ser modificados según las informaciones que nos lleguen en el curso de la acción y según los elementos aleatorios que sobrevendrán y perturbarán la acción. (Morin, 2007, p. 113)

Es decir, Morin (2007) identifica acción con estrategia. Aunado a ello, destaca que la estrategia no es estática, pues ofrece oportunidades para incorporar cambios en las acciones, dependiendo de que sus resultados se acerquen a o se alejen de los previstos o deseados. La misma idea se tiene en el Ministerio del Poder Popular para la Educación, donde dice que los proyectos "son considerados como una forma de organización del aprendizaje en la que maestros, maestras, estudiantes y familia buscan, en conjunto, solución a un problema de su interés, preferiblemente con relevancia social, mediante un proceso activo y participativo" (MPPE, 2007, p. 66). Esta búsqueda de solución por medio de un proceso activo involucra acciones que se modifican; tal como indica Ander-Egg (2007), al exponer que la planificación es un proceso que implica un ajuste y reajuste permanente entre medios, actividades y fines.

En síntesis, la planificación por proyectos está orientada a la acción ya que incluye estrategias, pero no es una estrategia. Además, se puede destacar que este docente posee un manejo teórico del tema, pero se contradice en la práctica, puesto que planifica por programa, lo cual, como vimos, es contrario a lo que plantea el MPPE (2007).

\section{Meta}

La planificación por proyectos la conciben algunos actores educativos participantes a modo de meta, idea que se puede evidenciar en las siguientes expresiones: $\mathrm{PH} 1$ dijo: “Un 
proyecto es una meta o propósito que uno quiere lograr", también D3 expresó: "Proyecto es una meta que queremos lograr, en la cual ejecutamos actividades planteadas"; asimismo, D1 señaló: "El proyecto es una meta que hay que cumplirla y debe ser compartida".

Sin embargo, Claret (2008) afirma que meta"es la expresión cuantitativa de lo que se pretende alcanzar con el proyecto, indicando cuándo y en qué tiempo se pretende alcanzar"(p. 27), así como el ámbito o espacio delimitado. También, para Malagón (2005), las metas"expresan lo que la comunidad quiere alcanzar en cuanto a actitudes y comportamiento (cultura), solución a problemas, necesidades y potencialidades, relacionadas con la planificación y la gestión de la institución educativa y de la proyección al futuro" (p. 277). De igual forma, para Senge (1995), "la meta representa aquello que la gente se compromete a lograr, a menudo al cabo de pocos meses" (p. 285).

En este sentido, la meta es el punto de llegada, es el fin hacia donde confluyen las acciones y se identifica con los objetivos o propósitos para dar respuesta a "qué hacer"y"qué se va a lograr" expresado de manera organizada en la planificación. Por consiguiente, un proyecto no es una meta, aunque sus objetivos apuntan a ella. Cabe destacar que una meta no es un objetivo, pero se puede entender como la expresión de un objetivo en términos cuantitativos y cualitativos; por eso, la realización de una meta supone la ejecución de una serie de actividades, las cuales comprenden la cantidad de trabajo específico que debe realizar un proyecto. En consecuencia, si las metas son como pequeñas acciones que se deben realizar para poder alcanzar al objetivo y si todo objetivo está compuesto por varias metas, que, unidas y alcanzadas lo conforman, un proyecto no es una meta. Antes bien, las acciones que lo configuran permiten lograr metas y objetivos previstos en él.

Resumiendo, se puede afirmar que los actores educativos conciben la planificación por proyecto como: visión, secuencia ordenada de pasos, acción colectiva, intención de logro, estrategia y meta. Al respecto es importante señalar que ninguno la definió como un proceso, algunos evidenciaron errores de conceptualización al confundir la planificación con algunos de sus pasos; muy pocos definieron planificación por proyectos, pues la mayoría se refirió a proyecto, pudiendo inferir que el proyecto no es visto como modo de planificar. Igualmente, muy pocos sujetos, que no eran docentes, señalaron su carácter participativo.

A continuación, se presenta la Tabla 1 que sintetiza los hallazgos de la investigación. En ese sentido, muestra las categorías que se generaron, las evidencias en las expresiones de los actores educativos y los autores que sirvieron de base para darles sustento teórico, o bien, confrontarlos. 
doi: http://dx.doi.org/10.15359/ree.21-2.13

URL: http://www.una.ac.cr/educare

CORREO: educare@una.cr

Tabla 1: Categorías construidas, sus evidencias y autorías para sustentar o confrontar

\begin{tabular}{|c|c|c|}
\hline Categorías & Expresiones de actores educativos & $\begin{array}{l}\text { Autorías para sustentar o } \\
\text { confrontar }\end{array}$ \\
\hline Visión & $\begin{array}{l}\text { D1: "Es una visión de lo que se quiere lograr. Es recopilar una } \\
\text { información para ejecutar algo que se quiere". } \\
\text { PH2: "El proyecto es una perspectiva o visión de lograr lo que uno } \\
\text { se propone, incluyendo el qué, cómo y cuándo lo voy a hacer". } \\
\text { PH4: "Un proyecto es una visión a futuro de actividades a } \\
\text { realizar para conseguir algo basado en una realidad social, } \\
\text { de la cual, salen unas variables y las que resulten, se les dará } \\
\text { respuestas a través de actividades que conforman un conjunto". }\end{array}$ & $\begin{array}{l}\text { - Armijo (2009) } \\
\text { - Ander-Egg y Aguilar (2000) } \\
\text { - Senge (1993) } \\
\text { - Senge (1995) } \\
\text { - MED (2004) }\end{array}$ \\
\hline $\begin{array}{l}\text { Secuencia } \\
\text { ordenada de } \\
\text { pasos }\end{array}$ & $\begin{array}{l}\text { PH2: "La planificación por proyectos son fases para lograr } \\
\text { metas, en las cuales se toman en cuenta las propuestas de los } \\
\text { involucrados en el proyecto" } \\
\text { DOC3: la "Planificación por proyectos es seguir unos pasos } \\
\text { para lograr todo lo plasmado en el proyecto" } \\
\text { DOC2: "La planificación por proyectos es la secuencia y el } \\
\text { orden de lo que se quiere lograr en los proyectos". }\end{array}$ & $\begin{array}{l}\text { - Collerette y Delisle (2001) } \\
\text { - Ander-Egg (2007) } \\
\text { - MPPE (2007) } \\
\text { - Rossi y Grimberg (2005) } \\
\text { - Molins (2007) } \\
\text { - Flores y Agudelo (2006) } \\
\text { - Pérez (2000) }\end{array}$ \\
\hline Acción colectiva & $\begin{array}{l}\text { REP1: "Un proyecto es cuando entre varias personas hacen } \\
\text { algo para beneficio de la comunidad donde viven" } \\
\text { E1 señaló: "Para mí un proyecto es un trabajo, cuando me } \\
\text { dicen: vamos a hacer algo, eso es un proyecto" } \\
\text { REP4 indicó: "La planificación por proyectos viene siendo la } \\
\text { reunión de docentes y estudiantes para los pasos que se van a } \\
\text { seguir en la ejecución de los proyectos". }\end{array}$ & $\begin{array}{l}\text { - Corredor (2010) } \\
\text { - Ander-Egg (2007) } \\
\text { - Ander-Egg y Aguilar (2000) } \\
\text { - MPPE (2007) }\end{array}$ \\
\hline Intención de logro & $\begin{array}{l}\text { D2: "el proyecto es para mí como un plan, es algo, son propuestas } \\
\text { a corto, mediano y largo plazo factibles de lograr" } \\
\text { PH1: "La planificación por proyecto es establecer un objetivo } \\
\text { por medio de etapas y diversas estrategias a aplicar y a ser } \\
\text { logradas a corto, mediano y largo plazo" } \\
\text { DOC3: "el proyecto es un documento para llevar a cabo unos } \\
\text { pasos a seguir, es la manera de trabajar ordenadamente". } \\
\text { REP2: "El proyecto es una propuesta para beneficio de la } \\
\text { escuela y la comunidad" } \\
\text { REP3: "Un proyecto es una solicitud de hacer algo para } \\
\text { beneficio de la escuela, para solucionar un problema" } \\
\text { E2: "Un proyecto es una propuesta para realizar algo a fin de } \\
\text { mejorar el funcionamiento de la escuela" } \\
\text { DOC2, señaló: "Un proyecto es una aspiración que uno quiere } \\
\text { lograr, que siempre se hace con un fin a futuro". }\end{array}$ & $\begin{array}{l}\cdot \text { Ander-Egg (2007) } \\
\text { Corredor (2010) } \\
\text {. Barrera }(2005) \\
\text { MPPE }(2007)\end{array}$ \\
\hline
\end{tabular}

continúa 


\begin{tabular}{|c|c|c|}
\hline Categorías & Expresiones de actores educativos & $\begin{array}{c}\text { Autorías para sustentar o } \\
\text { confrontar }\end{array}$ \\
\hline Estrategia & $\begin{array}{l}\text { PH3: “El proyecto es una estrategia que me va a permitir } \\
\text { dar solución a un problema o problemática; es la acción } \\
\text { de organizar un conjunto de actividades previstas para } \\
\text { dar solución a una problemática”Y agregó:"Yo no tengo } \\
\text { conocimiento de la planificación por proyectos porque yo } \\
\text { planifico con el programa”. }\end{array}$ & $\begin{array}{l}\text { Molins (2007) } \\
\text { Morin (2007) } \\
\text { MPPE (2007) } \\
\text { Ander-Egg (2007) }\end{array}$ \\
\hline Meta & $\begin{array}{l}\text { PH1:“Un proyecto es una meta o propósito que uno quiere lograr" } \\
\text { D3: "Proyecto es una meta que queremos lograr, en la cual } \\
\text { ejecutamos actividades planteadas" } \\
\text { D1: "El proyecto es una meta que hay que cumplirla y debe ser } \\
\text { compartida" }\end{array}$ & $\begin{array}{l}\text { Claret (2008) } \\
\text { Malagón (2005) } \\
\text { Senge (1995) }\end{array}$ \\
\hline $\begin{array}{l}\text { Desconocimiento } \\
\text { del PEIC }\end{array}$ & $\begin{array}{l}\text { DOC1: "Este año no hemos tenido el proyecto institucional" } \\
\text { PH2: "Pues no tengo conocimiento del PEIC de la escuela" } \\
\text { PH4 dijo:“Del proyecto institucional: no tengo conocimiento" } \\
\text { E1 señaló: "En cuanto al proyecto institucional, no sé nada" }\end{array}$ & $\begin{array}{l}\text { Rossi y Grinberg (2005) } \\
\text { MPPE (2007) }\end{array}$ \\
\hline
\end{tabular}

Nota: Elaboración propia de las autoras (2017)

\section{Implicaciones y conclusiones}

La planificación por proyectos es el componente curricular central del sistema educativo bolivariano. Su carácter participativo y colectivo alude a los procesos que escuela, familia y comunidad deben realizar con el fin de resolver problemas, formar ciudadanía participativa y organizar el aprendizaje en la escuela. Su concepción es holística, ya que orienta la comprensión contextual del proceso de planificación, de protagonistas y de sus contextos, facilitando la comunicación e interacción entre los diferentes actores sociales y educativos. Ya que toda interacción humana involucra unos valores, se enfatiza en los valores presentes en la Constitución de la República Bolivariana de Venezuela del 20 de diciembre de 1999 (2000): libertad, igualdad, justicia, paz, independencia, solidaridad, bien común, integridad territorial, convivencia y ciudadanía. Estos valores deben estar insertos en cada proyecto que se elabore con el fin de formar una ciudadanía integral para una existencia digna y provechosa del estudiantado y la comunidad.

En lo esencial, el conocimiento de la planificación por proyectos facilita que los actores educativos tengan la oportunidad de desarrollar acciones que permitan alcanzar el futuro deseado. Igualmente, ayuda a combatir ciertos obstáculos a la participación protagónica en la transformación de su entorno, tales como: la falta de vinculación en la construcción colectiva de acuerdos, el distanciamiento de la realidad y la evasión del compromiso, pues este surge de la participación en su construcción. En este contexto, considerando que las creencias y concepciones inciden y guían nuestro actuar, se tuvo como objetivo derivar implicaciones a 
doi: http://dx.doi.org/10.15359/ree.21-2.13

URL: http://www.una.ac.cr/educare

CORREO: educare@una.cr

partir de las concepciones sobre planificación por proyectos que poseen los actores educativos, concepciones recabadas por medio de una entrevista cualitativa con el fin de obtener descripciones de sus creencias y experiencias al respecto.

Los resultados obtenidos indican que los actores educativos conciben la planificación por proyectos como visión, secuencia ordenada de pasos, acción colectiva, intención de logro, estrategia y meta. Cabe destacar que nadie definió la planificación por proyectos como planificación ni como proceso. Por el contrario, sus nociones refieren, por una parte, a lo que se quiere lograr: visión, intención de logro, meta. Por la otra, la menos, a la acción, la cual puede ser colectiva o una estrategia para resolver problemas; ambas concepciones acordes con los planteamientos del MPPE $(2007,2012)$, pero que fueron declaradas por muy pocos representantes, un estudiante y un docente. Esto dejó entrever que, aparentemente, no existe la debida participación en la elaboración y ejecución de los proyectos educativos. Por último, es importante resaltar la concepción como "secuencia ordenada de pasos", pues, como se dijo, ninguno la describió por medio del término "proceso".

El Ministerio del Poder Popular para la Educación en el Diseño Curricular Bolivariano entiende la educación como un proceso social que se crea en colectivo y emerge de las raíces de cada pueblo. A tal fin asume la concepción educativa de Freire como proceso que propicia a estudiantes y docentes el aprender '"... a leer la realidad para escribir su historia', lo cual supone comprender críticamente su mundo y actuar para transformarlo" (MPPE, 2007, p. 49), en concordancia, la acción educativa se debe realizar mediante la planificación por proyectos educativos.

Con la misma intención, el Ministerio de Educación de Chile (MINEDUC, División de Educación General, 2016) elaboró un documento con orientaciones para realizar la planificación del año escolar 2016 amparado en las definiciones instituidas en el proyecto educativo institucional (PEI) donde cada comunidad educativa expresa sus sentidos y fines. Dichas orientaciones tienen el propósito de optimizar el uso de tiempo, los recursos educativos y las acciones pedagógicas para promover el desarrollo de aprendizajes efectivos en todos sus estudiantes.

Sin embargo, aún cuando en estudios y documentos diferentes se expone la importancia de planificar por proyectos, en las entrevistas se encontró que los actores educativos:

- No ven los proyectos como un modo de planificación. Ante la pregunta por la planificación por proyectos, la mayoría respondió definiendo proyecto.

- El estudiantado aprenderá contenidos y habilidades no necesariamente vinculados con su realidad ni con la solución de problemas de la comunidad.

- Será difícil que los actores educativos en general vinculen la educación con la vida diaria o lean la realidad y sean capaces de transformarla. 
- Con estas concepciones y desde ellas, poco se enseña en pro de potenciar el desarrollo comunitario, puesto que la comunidad no está incluida en la elaboración de los proyectos educativos, trayendo como consecuencia que pocos podrán decir que el proceso de enseñanza aprendizaje ocurre en relación con el contexto histórico social de la comunidad.

Siendo así, es importante preguntarse y reflexionar si realmente el personal docente esta conformado por agentes activadores, mediadores de los saberes, del sentir, del hacer social y cultural y del proceso de apropiación de los aprendizajes por parte de sus estudiantes. Asimismo, sobre el papel social y educativo que cumplen padres, madres, representantes y comunidad en general respecto de la apropiación del saber popular en vínculo estrecho con el conocimiento académico y cientííco.

La epistemología se asocia con conocimiento, enfatiza el reconocimiento o reflexión del conocer sobre el sujeto y el objeto, la realidad yel pensamiento. EI MPPE (2007), en las orientaciones epistemológicas, establece que la formación de la nueva ciudadanía responsable considera un aprendizaje inter y transdisciplinario al integrar las diferentes áreas del conocimiento y los saberes populares por medio de experiencias colectivas y contextualizadas. En ese sentido, los temas de aprendizaje deben surgir de la relación del estudiantado con su entorno y la investigación en escenarios reales, proceso que debe tener lugar mediante la planificación por proyectos de aprendizaje. Estos constituyen la base de la construcción de conocimientos estudiantiles y su validación en la práctica al tomar en cuenta el contexto histórico y cultural, los saberes y sentires del pueblo mediante el diálogo, en una relación horizontal, dialéctica, de reflexión crítica, dejando de lado procesos de construcción de saberes individualistas y fragmentados.

En ese sentido, Barrios y Chaves (2015, p. 13) argumentan que en Colombia "las políticas educativas desde el periodo colonial hasta el siglo XXI, están bajo la influencia de los ideales ilustrados de Europa", por lo que proponen el modelo pedagógico enseñanza para la comprensión $(\mathrm{EpC})$ y los proyectos de aula, ya que estos permiten desarrollar competencias cognoscitivas de diferente índole en el estudiantado que le permiten incidir en espacios familiares y comunitarios.

Sin embargo, en este estudio los pocos actores educativos que manifestaron su concepción de proyectos como acción colectiva fueron dos representantes y un estudiante. Es decir, ninguna persona docente expresó este significado; no obstante ser quienes, supuestamente, elaboran el proyecto de aprendizaje en unión con los actores sociales de la comunidad donde se encuentra ubicada la escuela. Luego, es posible realizar algunas inferencias con respecto al conocimiento que construirán quienes estudian:

- Aprenderán contenidos y construirán conocimientos descontextualizados, pues su contexto, su comunidad, no está involucrada en la elaboración de los proyectos de aprendizaje. Por eso, difícilmente construirán un conocimiento socializado, un saber validado en la práctica y dentro de un contexto histórico y cultural que incluya los saberes y sentires del pueblo. 
doi: http://dx.doi.org/10.15359/ree.21-2.13

URL: http://www.una.ac.cr/educare

CORREO: educare@una.cr

- Tampoco se podrá hablar de un diálogo de saberes, si los contenidos de aprendizaje que conforman el proyecto no incluyen los saberes populares respecto del tema tratado.

- Como resultado de lo anterior, poco aprendizaje inter y transdisciplinario se logrará ya que no se integran las áreas del conocimiento ni los saberes populares. De hecho, todavía hay docentes que planifican por programas (se entiende que de su asignatura, pues lo dijo un profesor por horas), luego, no existe tal interdisciplinariedad. En consecuencia, se sigue planificando y realizando el proceso educativo con un aprendizaje fragmentado, alejado de la realidad del estudiante y contrario a las políticas educativas emanadas del sistema educativo bolivariano.

En lo que se refiere al aspecto político, es importante señalar que las políticas constituyen el marco referencial para la toma de decisiones. Al respecto, Ander-Egg (2007) expresa que toda planificación "es la instrumentación de un proyecto político" (p. 40). En este orden de ideas, el MPPE (2007) declara la educación como proceso social, enraizado en cada pueblo. En tanto y cuanto acto político, expresa procesos sociales, culturales y educativos cuya finalidad es impulsar el pensamiento liberador, creador y transformador, la reflexión crítica, la participación ciudadana y los sentimientos de honor, probidad, amor a la patria, a las leyes y al trabajo.

Para ello, el mismo documento establece que la organización y estructuración de los diferentes elementos que condicionan el proceso de aprendizaje se deben realizar por medio de la clase, el proyecto y el plan integral. De esta forma, la planificación del aprendizaje por proyectos supone, en medio del actual contexto político-social, la necesidad de formar una nueva ciudadanía con conciencia social, comprometida con el desarrollo de las políticas sociales y garante de su adecuada implementación, mediante el ejercicio efectivo de su papel de contralora social. En síntesis, envuelve el actuar en colectivo desde la educación y sociedad para la transformación social.

No obstante, al retomar las concepciones sobre la planificación por proyectos que asumen los actores educativos responsables de cumplir y hacer cumplir tales políticas educativas, nos enfrentamos a una realidad donde esto no será posible, considerando que, por una parte, las concepciones de visión, meta, intención de logro expresan lo que se quiere lograr, solo propósitos, mas no revelan las acciones. Por otra parte, una planificación por proyectos entendida como acción colectiva no está en la mente del personal docente; además, un solo docente indicó que es una estrategia (entendida como acción) para solucionar problemas. Aunado a lo anterior, la noción de la planificación como proceso está implícita, pues ninguno de los actores educativos la mencionó, siempre la referencia fue a secuencia ordenada de pasos, fases, organización. Tampoco se hizo mención de lo colectivo, de la participación de la comunidad en el proceso de elaboración de los proyectos educativos. En consecuencia, nos permite inferir que en la planificación por proyectos: 
- Prevalece la ausencia de la noción de una educación como proceso social, colectivo y enraizado en cada pueblo.

- Predomina una finalidad orientada a la enseñanza y aprendizaje de contenidos y difícilmente a fomentar el pensamiento liberador, creador y transformador, la reflexión crítica, la participación ciudadana para formar un ser humano integral.

- Se omite la formación para la participación democrática, protagónica y corresponsable, en oposición a una democracia representativa. Es decir, no se aprende a participar tal como preceptúa el pilar de aprender a convivir y participar, evidente en el hecho que algunos actores manifestaron su desconocimiento sobre el Proyecto Educativo Integral Comunitario (PEIC), el cual, como proyecto institucional, debe involucrar a toda la comunidad en su elaboración. Luego, es posible inferir que este proyecto no se realiza de manera participativa y, por lo tanto, no involucra a todos los actores educativos y sociales que conforman la comunidad. En consecuencia, pocos aprenderán a participar en la sociedad como ciudadanos y ciudadanas responsables.

- Corroborando lo anterior, pocos actores: 2 representantes y 1 estudiante (17,65\%), entienden la planificación por proyectos como una acción colectiva; es decir, implican la participación, el trabajo conjunto; concepción que nadie del personal docente comparte. Luego, si educar para la participación requiere de formación (se puede/ debe enseñar) y se aprende fundamentalmente con el ejemplo y las acciones, ¿cómo la aprenderán estos estudiantes? Nadie del personal docente entrevistado entiende así la planificación, sumado a que se sabe que nuestras creencias y concepciones guían nuestras acciones. Luego, en este proceso educativo el estudiantado difícilmente aprenderá a participar como ciudadanos y ciudadanas y tampoco se educa a la comunidad para la participación.

En resumen, si la participación no se enseña con el ejemplo, queda en entredicho la nueva ciudadanía de un educando con conciencia social, comprometido con el desarrollo de las políticas sociales y garante de su adecuada implementación. En consecuencia, parece que en la realidad no se está formando la nueva ciudadanía que establece el Currículo Nacional Bolivariano (MPPE, 2007).

Por último, es de resaltar que la educación pretende fomentar el pensamiento liberador, creador y transformador, lo que nos lleva a vislumbrar las posibles implicaciones para el cambio. Planificar es un modo de actuar sobre la realidad que involucra dos lógicas: una refiere a la elaboración del plan en tanto que documento contentivo de la planificación elaborada y, la otra, al proceso de planificación y ejecución de lo planificado. La planificación como proceso y estrategia quiere decir actuar en el presente con miras a lograr el futuro deseado, es decir, cambiar. 
doi: http://dx.doi.org/10.15359/ree.21-2.13

URL: http://www.una.ac.cr/educare

CORREO: educare@una.cr

En ese orden de ideas, Aguerrondo y Vaillant (2015) sugieren la necesidad de una mutación, un cambio de paradigma permanente en la educación, que permita superar la exclusión educativa, así como los problemas que aquejan la educación en Latinoamérica. Reconocen que este cambio no es fácil por su complejidad y recogen varias experiencias exitosas en diferentes países latinoamericanos. La experiencia que viene al caso es el modelo de aprendizaje en servicio (APS). Indican las autoras:

Este modelo, si bien no es originario de la región, se adapta plenamente a sus características, como lo prueba su importante expansión en países latinoamericanos. Aprendizaje en Servicio es una metodología pedagógica que promueve el desarrollo de competencias a través de actividades escolares de servicio a la comunidad. (Aguerrondo y Vaillant, 2015 p. 69)

Por ende, al considerar las necesidades de la comunidad permite el aprendizaje de nuevos conocimientos y el desarrollo personal; lo cual exige una participación activa del alumnado y una cuidadosa planificación y organización de las actividades. Pero si, como se encontró, los actores educativos conciben la planificación y los proyectos como propuestas de acción o ideas de logro, expresarán buenas intenciones mas no acciones concretas para realizar, especialmente si tales intenciones son declaradas por el personal docente, tomando en cuenta que tales personas tienen la responsabilidad de educar a las nuevas generaciones para la participación, para la acción y el cambio.

Es importante señalar que solamente tres personas definieron los proyectos con una perspectiva de acción colectiva, pero fueron dos representantes y un estudiante, actores educativos quienes no tienen en sus manos la responsabilidad ni las herramientas para enseñar y lograr los cambios que se requieren desde procesos de enseñanza aprendizaje. En consecuencia, podemos afirmar que las concepciones de planificación por proyectos que poseen los actores educativos entrevistados no implican la generación de cambios. Como muestra, basta señalar que aún hay al menos una persona docente que no planifica por proyectos y afirma seguir un programa; o sea, él mismo se resiste al cambio.

En síntesis, ya que el ser humano actúa desde sus concepciones y creencias, sus modos de entender la planificación por proyectos obstaculizan un proceso educativo que pretende una educación emancipadora, liberadora y dignificante, enmarcada en la Constitución y cuya finalidad es formar una nueva ciudadanía republicana, con autonomía creadora, transformadora y con ideas revolucionarias; así como una actitud emprendedora para poner en práctica nuevas y originales soluciones en la transformación endógena del contexto social-comunitario.

Seconcluye, entonces, que las concepciones de los actores educativos sobre la planificación por proyectos no conducen a las acciones para el logro de los fines educativos establecidos, pues dificultan una educación para la democracia participativa, para la acción y el cambio, limitando 
la formación de la nueva ciudadanía responsable y cooperativa. Ello implica que el estudiantado no se está formando para ser protagonista de la nueva ciudadanía ni se está garantizando su completo desarrollo, tanto individual como colectivo. Se sugiere, en principio, formar para la participación al personal docente como responsable y garante del proceso educativo.

\section{Referencias}

Aguerrondo, I. y Vaillant, D. (2015). El aprendizaje bajo la lupa: Nuevas perspectivas para América Latina y el Caribe. Panamá: UNICEF. Recuperado de https://www.unicef.org/lac/UNICEF Aprendizaje bajo la lupa nov2015(1).pdf

Ander-Egg, E. (2007). Introducción a la planificación estratégica. Buenos Aires: Lumen Hvmanitas.

Ander-Egg, E. y Aguilar, M. J. (2000). Cómo elaborar un proyecto. Guía para diseñar proyectos sociales y culturales (15a ed.). Buenos Aires: Lumen Hvmanitas.

Armijo, M. (2009). Manual de planificación estratégica e indicadores de desempeño en el sector público (Versión preliminar). Santiago, Chile: ILPES/CEPAL. Recuperado de http://www. cepal.org/ilpes/noticias/paginas/3/38453/manual_planificacion estrategica.pdf

Barrera, M. F. (2005). Planificación prospectiva y holística (4a ed.). Caracas: Quirón Ediciones.

Barrios, L. y Chaves, M. (2015). El proyecto de aula como estrategia didáctica en el marco del modelo pedagógico enseñanza para la comprensión. Experiencia del Colegio Visión Mundial en comunidades vulnerables de Montería (Córdoba-Colombia). En J. Asenjo, Ó. Macías y J. C. Toscano (Coords.), Memorias del Congreso Iberoamericano de Ciencia, Tecnología, Innovación y Educación (pp. 1-21). Buenos Aires: OEl. Recuperado de www.oei. es/historico/congreso2014/memoriactei/209.pdf

Castellano, H. (2006). La planificación del desarrollo sostenible. Contenidos, entorno y métodos. Caracas: UCV.

Claret, A. (2008). Proyecto comunitario e investigación cualitativa. Sexta edición. Caracas, Venezuela: Texto.

Collerette, P. y Delisle, G. (2001). La planificación del cambio: Estrategias de adaptación para las organizaciones. México: Trillas.

Constitución de la República Bolivariana de Venezuela del 20 de diciembre de 1999. (24 de marzo del 2000). Gaceta Oficial, № 5453, Extraordinario.

Corredor, J. (2010). La planificación. Nuevos enfoques y proposiciones para su aplicación en el siglo XXI. Caracas: Vadell Hermanos Editores. 
doi: http://dx.doi.org/10.15359/ree.21-2.13

URL: http://www.una.ac.cr/educare

CORREO: educare@una.cr

Elliott, J. (1996). El cambio educativo desde la investigación-acción (Trad. P. Manzano, 2a ed.). Madrid: Morata.

Flores, H. y Agudelo, A. (2006). La planificación por proyectos. Una estrategia efectiva para enseñar y aprender (Colección Brújula Pedagógica). Caracas: El Nacional.

Kemmis, W. (1996). Mejorando la educación mediante la investigación social. México: Trillas.

Malagón, F. A. (2005). Planificación institucional en la educación. Un reto estratégico de cambio para los gerentes educativos innovadores. Bogotá: Panamericana.

Matos, Y., Pasek, E. y Rojas, A. (2014). Actividades para favorecer la participación de los padres y representantes en el preescolar. Revista Electrónica Sustentabilidad al Día, 1, 1-21.

Ministerio de Educación (MINEDUC), División de Educación General. (2016). Orientaciones. Jornada de planificación establecimientos educacionales. Santiago de Chile: Autor. Recuperado de http://www.mineduc.cl/wp-content/uploads/sites/19/2016/01/ Orientaciones-J-de-planificaci\%C3\%B3n-1-y-2-Marzo-EE..pdf

Ministerio de Educación y Deportes (MED). (2004). Escuelas bolivarianas. Avance cualitativo del proyecto. Caracas. Caracas: Autor.

Ministerio del Poder Popular para la Educación (MPPE). (2007). Currículo nacional bolivariano. Diseño curricular del sistema educativo bolivariano. Caracas: Autor.

Ministerio del Poder Popular para la Educación (MPPE). (2012). La planificación educativa en el subsistema de educación básica. Caracas: Autor. Recuperado de https://docs.google.com/ file/d/0B1bDSdW6P6teLXI5aHQyNFc3YVU/edit

Molins, M. (2007). Teoría de la planificación. Caracas: Universidad Central de Venezuela.

Morin, E. (2000). La complejidad y la acción. Recuperado de http://www.utp.edu.co/rectoria/ documentos/la-complejidad-y-la-accion-edgar-morin

Morin, E. (2007). Introducción al pensamiento complejo. Barcelona: Gedisa.

Pasekde Pinto, E., Ávila deVanegas, N.y Matos de Rojas,Y.(2015).Concepciones sobre participación social que poseen los actores educativos y sus implicaciones. Revista Paradigma, 35(2), 99-121. Recuperado de http://revistas.upel.edu.ve/index.php/paradigma/article/view/ 3017/1429

Pazos, C. (Febrero, 2001). Planificación de proyectos socio-educativos: Una revisión de aspectos críticos y algunos aprendizajes a partir de la práctica. En III Congreso "La atención a la diversidad en el sistema educativo". España: Universidad de Salamanca, INICO. Recuperado de https://campus.usal.es/ inico/actividades/actasuruguay2001/3.pdf 
Pérez, G. (2000). Elaboración de proyectos sociales. Casos prácticos. Madrid: Narcea.

Rodríguez, G., Gil, J. y García, E. (1999). Metodología de la investigación cualitativa (2a ed.). Granada, España: Aljibe.

Rossi, M. y Grinberg, M. (2005). Proyecto educativo institucional. Acuerdos para hacer escuelas. Buenos Aires: Magisterio Río de la Plata.

Sarceda, M., Seijas, S., Fernández, V. y Fouce, D. (2015). El trabajo por proyectos en educación infantil: Aproximación teórica y práctica. RELAdEI. Revista Latinoamericana de Educación Infantil, 4(3), 159-176. Recuperado de http://redaberta.usc.es/reladei/index.php/reladei/ article/download/271/218

Secretaría de Educación Pública (SEP). (2009). Modelo de gestión educativa estratégica. Programa escuelas de calidad. México: Autor. Recuperado de http://upnmorelos.edu.mx/2013/ documentos descarga 2013/fuentes informacionMEB/MEB064\%20MGEE.pdf

Senge, P. (1993). La quinta disciplina. Barcelona: Granica.

Senge, P. (1995). La quinta disciplina en la práctica. Estrategias y herramientas para construir la organización abierta al aprendizaje. Barcelona: Granica.

Taylor, S. J. y Bogdan, R. (1992). Introducción a los métodos cualitativos de investigación. La búsqueda de significados. Buenos Aires: Editorial Paidós. 